\title{
ESTRESSE POR ALUMÍNIO E POR ACIDEZ EM CULTIVARES DE SOJA
}

\author{
Ceci Castilho Custódio*; Daniel Carlini Bomfim²; Sérgio Marques Saturnino²; Nelson Barbosa \\ Machado Neto ${ }^{1}$ \\ ${ }^{1}$ Depto. de Biologia Vegetal e Fitossanidade - UNOESTE, Rod. Raposo Tavares km 572, Bairro Limoeiro - \\ CEP: 19067-175 - Presidente Prudente, SP. \\ ${ }^{2}$ Graduandos em Engenharia Agronômica - UNOESTE. \\ *Autor correspondente <cecicc@ig.com.br>
}

\begin{abstract}
RESUMO: Os solos tropicais e subtropicais são normalmente ácidos. O objetivo deste trabalho foi estudar o comportamento de quatro cultivares de soja com relação a germinação e vigor das plântulas quando submetidos a concentrações de alumínio $\left(0 ; 0,25 ; 0,5 ; 1,0\right.$ e $\left.1,5 \mathrm{mmol}_{\mathrm{c}} \mathrm{dm}^{-3} \mathrm{de}^{3^{3+}}\right)$ e de pH $(7,0 ; 6,0 ; 5,5 ; 5,0$ e 4,5). As concentrações de alumínio não afetaram a germinação e o peso seco de raiz, em contrapartida o comprimento de hipocótilo e de raiz , o peso seco da parte aérea e a classificação de vigor foram afetados. Na faixa entre 0,25 e $1,0 \mathrm{mmol}_{\mathrm{c}} \mathrm{dm}^{-3}$ ocorreu estímulo para o desenvolvimento da plântula (comprimento de hipocótilo e raiz). Estatisticamente, o comprimento de raiz teve seu maior desenvolvimento no tratamento de $1,0 \mathrm{mmol}_{\mathrm{c}}$ $\mathrm{dm}^{-3} \mathrm{Al}^{3+}$. Os cultivares 'Xingu', 'Pintado' e 'Pioneira' se destacaram em todos os tratamentos. Os tratamentos de $\mathrm{pH}$ afetaram a germinação, o comprimento de hipocótilo e de raiz, o peso seco de parte aérea e de raiz e a classificação do vigor. A germinação foi maior no $\mathrm{pH}$ 6,0 enquanto que o $\mathrm{pH}$ 7,0 produziu maior comprimento de hipocótilo, de raiz, maior peso seco de parte aérea e de raiz. Os cultivares 'Xingu', 'Pioneira' e 'Pintado' se destacaram por apresentarem as maiores médias de germinação mesmo nos tratamentos com maior acidez, indicando que este caráter foi pouco afetado pelos tratamentos. O cultivar Conquista foi o mais sensível aos tratamentos de alta acidez.
\end{abstract}

Palavras-chave: Glycine max, tolerância, germinação, vigor

\section{ALUMINUM AND ACIDITY STRESS ON SOYBEAN CULTIVARS}

\begin{abstract}
Tropical and subtropical soils are frequently acid. In this paper four soybean varieties were contrasted in relation to germination and vigour of soybean (Glycine max L. Merril.) seedlings when seeds were submitted to aluminium concentrations $\left(0 ; 0.25 ; 0.5 ; 1.0\right.$ and $\left.1.5 \mathrm{mmol}_{\mathrm{c}} \mathrm{dm}^{-3} \mathrm{Al}^{3+}\right)$ and $\mathrm{pH}$ values $(7.0$; $6.0 ; 5.5 ; 5.0$ and 4.5). The concentrations of aluminum did not affect germination and root dry weight, however, hypocotyl and root length, shoot dry weight and seedling vigour classification were affected. In the range between 0.25 and $1 \mathrm{mmol}_{\mathrm{c}} \mathrm{dm}^{-3}$ there was an improvement of the development of the seedlings (hypocotyl and root length). Root length had its greatest development at $1.0 \mathrm{mmol}_{\mathrm{c}} \mathrm{dm}^{-3} \mathrm{Al}^{3+}$. Cultivars 'Xingu', 'Pintado' and 'Pioneira' presented the best results for all treatments. The $\mathrm{pH}$ treatments affected the germination, hypocotyl and root length, shoot and root dry weights and seedling vigour classification. The germination was superior at $\mathrm{pH} 6.0$ while at $\mathrm{pH} 7.0$ produced longer hypocotyls and roots, shoot and root dry weights. Cultivars 'Xingu', 'Pioneira' and 'Pintado' had the highest germination even in the most acid treatments, indicating that germination is not severely affected by the acidity of the substratum. The 'Conquista' was the most sensitive high acidity cultivar.

Key words: Glycine max, tolerance, germination, vigour
\end{abstract}

\section{INTRODUÇÃO}

Os solos tropicais e subtropicais são normalmente ácidos, seja pela ocorrência de precipitação suficientemente alta para lixiviar quantidades apreciáveis de bases permutáveis do solo, seja pela ausência de minerais primários e secundários, responsáveis pela reposição dessas bases. Sendo assim, devido a grandes áreas de solos ácidos aráveis, a importância da acidez, sob o aspecto prático, sobrepuja a da alcalinidade.

Além da ocorrência natural da acidez, o próprio cultivo tende a acentuar o problema, principalmente devido a absorção de cátions pelas raízes das plantas, deixando em seus lugares quantidades equivalentes de íons de hidrogênio (Vitti \& Casarin, 1992).
Em solos com pH excessivamente baixo ocorre a diminuição na disponibilidade de nutrientes como fósforo, cálcio, magnésio, potássio e molibdênio, e aumento da solubilização de íons como zinco, cobre, ferro, manganês e alumínio que, dependendo do manejo do solo e da adubação utilizados, podem atingir níveis tóxicos às plantas (Brasil, 1998). Rutty et al.(1995) utilizando plantas jovens de soja crescidas em pH 4,2 e expostas a concentrações de $\mathrm{Al}^{3+}$, observaram que acima de $10 \mathrm{mmol} \mathrm{m}^{-3}$ ocorreu um estímulo a absorção de $\mathrm{NO}_{3}^{-}$, enquanto que acima de $44 \mathrm{mmol} \mathrm{m}^{-3}$ ocorreu decréscimo na absorção de $\mathrm{NO}_{3}^{-}$. Onde ocorreu estímulo foi observado elongação da raiz, o que não ocorreu a altas concentrações de $\mathrm{Al}^{3+}$. A diminuição da elongação foi muito mais severa do que a inibição da absorção de $\mathrm{NO}_{3}{ }^{-}$ 
sugerindo que altas concentrações de $\mathrm{Al}^{3+}$ inibem $\mathrm{o}$ crescimento radicular, mas não afetam o suprimento de nitrogênio.

Obroucheva \& Antipova (1994) fizeram um estudo sobre o fenômeno de crescimento em ambiente ácido em sementes de Vicia faba, durante as fases iniciais da embebição e germinação. A extrusão da raiz primária foi precedida por liberação de prótons para o ambiente, conhecido como padrão de crescimento ácido. O uso de tampão ácido promoveu a iniciação da elongação de células e protrusão da raiz primária. A extrusão de prótons pela ATPase, para a parede celular aumentou a plasticidade da mesma, facilitando a iniciação da elongação das células e consequentemente o início visível da germinação.

Buerkert \& Marschener (1992) montaram um experimento em câmara de crescimento com Phaseolus vulgaris cv. 'Negro de Chiapas' e 'Negro Huasteco' para investigar a diminuição da infecção de fungos com aplicação de cálcio. Os resultados indicaram que o pH e a relação $\mathrm{Ca}^{++} / \mathrm{Al}^{3+}$ na solução do solo ao redor das sementes determinaram seu padrão de crescimento e absorção de solutos durante a germinação. Quanto maior o $\mathrm{pH}$ e $\mathrm{Ca}^{++}$e menor $\mathrm{Al}^{3+}$ ao redor das sementes maior foi a lixiviação de aminoácidos e menor a de carboidratos. A diminuição da infecção por fungos com a aplicação de $\mathrm{Ca}^{++}$ocorreu devido ao decréscimo na quantidade de lixiviados para o desenvolvimento do fungo, principalmente carboidratos.

Embora os campos agrícolas ácidos sejam corrigidos com calcário, esta correção se dá numa estreita faixa superficial, o que deixa uma camada subsuperficial ácida e com alumínio tóxico. Bushamuka \& Zobel (1998) trabalharam com solos depositados em camadas, de solo não corrigido ( $\mathrm{pH}$ 4,5 e saturação de alumínio $92 \%$ ) sob solo corrigido, afim de observarem o desenvolvimento radicular de milho e soja. Três tipos de raízes foram observados, raízes laterais, superficiais e de profundidade, sendo que cultivares sensíveis apresentaram crescimento radicular apenas na camada corrigida. Beyrouty et al. (2000) ressaltam que, em soja, os problemas de crescimento radicular reduzido podem comprometer a produção, principalmente se, concomitantemente, houver problemas com disponibilidade hídrica. Spehar \& Makita (1994) separaram cultivares, com resistência ao alumínio utilizando-se de faixas de 1,5 e 3 ppm de $\mathrm{Al}^{3+}(0,17$ e $0,34 \mathrm{mmol}_{\mathrm{c}} \mathrm{dm}^{-3} \mathrm{Al}^{3+}$ ), com base no comprimento radicular, reputando ser esta uma medida simples, de curta duração, baixo custo e principalmente não destrutiva.

O alumínio trocável dos solos, além de interferir no desenvolvimento da planta pode reduzir a germinação. Souza Filho \& Dutra (1998) mostraram que a germinação de sementes de Calopogonium mucunoides em solos com concentração de alumínio de $20 \mathrm{mmol}_{\mathrm{c}} \mathrm{dm}^{-3}$ foi $6 \%$ menor que o controle. Em concentrações inferiores não ocorreram prejuízos à germinação. Já Szymanska \& Molas (1996) não encontraram efeito do $\mathrm{Al}^{3+}$ na germinação de Cucumis sativus. Porém, as concentrações de $\mathrm{Al}^{3+}$ influenciaram significativamente no crescimento. Em concentrações de 1 a $5 \mathrm{mg} \mathrm{dm}^{-3}$ de $\mathrm{Al}^{3+}$ o crescimento da plântula foi estimulado, não acarretando anormalidades morfológicas ou de desenvolvimento, porém em concentrações de 20 a $40 \mathrm{mg} \mathrm{dm}^{-3}$ de $\mathrm{Al}^{3+}$ ocorreu inibição do crescimento de plântulas e danos nas mesmas.

Fageria \& Baligar (1999) trabalhando com várias culturas em casa de vegetação utilizando-se solos com $\mathrm{pH}$ variando de 4,9 a 7,0 , notaram que todas as culturas tiveram o peso de matéria seca da parte aérea afetado pelo $\mathrm{pH}$, e em soja o valor ideal foi de 5,6. Todavia, Borkert \& Cox (1999) notaram que com a diminuição de $\mathrm{pH}$, numa faixa de 5,0 a 7,0 o peso seco de plantas de soja decresceu com o $\mathrm{pH}$.

Os cultivares podem apresentar diferenças quando submetidos a condições de baixo $\mathrm{pH}$. Cultivares com maior absorção de nutrientes tendem a ser mais tolerantes a condições ácidas. Segundo Blaha et al. (1997) o uso de seleção na fase juvenil para estas condições de desenvolvimento parece ser favorável. Chukwuma \& Tiwari (1995) estudaram 85 linhas de soja quanto a eficiência produtiva em baixo $\mathrm{pH}$ de solos (entre 5,0 e 5,5) quando comparados ao $\mathrm{pH} 6,8-7,0$. Os cultivares foram separados baseados na relação P/Al e índice de colheita. Após a avaliação em ensaios de crescimento em casa de vegetação, por 35 dias, e em campo até a colheita, concluíram que 22 cultivares eram altamente resistentes a baixo $\mathrm{pH}$ de solo.

O objetivo deste trabalho foi estudar o comportamento de quatro cultivares de soja com relação a germinação e vigor das plântulas quando submetidos a estresse ácido por alumínio ou pH.

\section{MATERIAL E MÉTODOS}

Foram utilizados quatro cultivares de soja, 'Pioneira' (MT/BR 49), 'Conquista' (MG/BR 46), 'Pintado' (MT/BRS 63) e 'Xingu' (MT/BR 51), colhidas na safra 98/ 99 e doadas pela empresa Sementes Maggi Ltda., localizada no município de Sapezal - MT (13³2'48" S e $\left.58^{\circ} 48^{\prime} 55^{\prime \prime} \mathrm{W}\right)$.

O experimento foi conduzido no período de agosto a outubro de 1999 no Laboratório de Análise de Sementes da Faculdade de Ciências Agrárias da Universidade do Oeste Paulista - UNOESTE, localizada em Presidente Prudente - SP.

Assim que as sementes foram recebidas passaram por processo de limpeza manual, retirando-se do lote as sementes quebradas, trincadas, rachadas, enrugadas, restando apenas as sementes puras e externamente perfeitas. Após esse processo as sementes foram mantidas, a $15^{\circ} \mathrm{C}$, na sala de armazenamento de sementes do laboratório. 
Os lotes de sementes foram submetidos a germinação em diferentes concentrações de tricloreto de alumínio $\left(\mathrm{AlCl}_{3}\right)$ e ácido clorídrico $(\mathrm{HCl})$ que proporcionaram às soluções um efeito de estresse ácido pela presença de $\mathrm{Al}^{3+}$ e de $\mathrm{H}^{+}$, respectivamente. Assim sendo, foram utilizados diferentes doses de tricloreto de alumínio para obtenção de soluções com as seguintes concentrações $0 ; 0,25 ; 0,5 ; 1,0$ e $1,5 \mathrm{mmol}_{c} \mathrm{dm}^{-3} \mathrm{de} \mathrm{Al}^{3+}$. Para $\mathrm{pH}$ as soluções foram obtidas corrigindo-se a água destilada com $\mathrm{HCl} 0,001 \mathrm{~N}$ até a obtenção do $\mathrm{pH}$ desejado de 7,$0 ; 6,0 ; 5,5 ; 5,0$ e 4,5 .

$O$ teste de germinação consistiu em se colocar quatro repetições de cinqüenta sementes, envolvidas entre três folhas embebidas com 2,25 vezes o seu peso com as soluções mencionadas anteriormente (Krzyzanowski, 1991).

Uma vez colocadas as sementes nas toalhas de papel, estas foram enroladas formando um cilindro, os quais foram envolvidos em sacos plásticos de polietileno e conduzidos até a sala de germinação, sendo mantidos em um germinador, tipo Mangesldorf, a temperatura constante de $25^{\circ} \mathrm{C}$. As contagens foram feitas aos 5 e 8 dias, computando-se as plântulas normais, anormais e as sementes mortas (Brasil, 1992). As plântulas normais foram classificadas, para avaliação do vigor, em normais fortes e fracas, de acordo com Nakagawa (1999). Os resultados foram expressos em porcentagem.

Para avaliação do desempenho das plântulas foram utilizadas quatro repetições de 10 sementes semeadas em toalhas de papel para germinação formando uma única linha de sementes a $10 \mathrm{~cm}$ da borda superior da folha. Estas foram submetidas as mesmas condições do teste de germinação sendo os dados obtidos após oito dias. Obtiveram-se destes materiais dados de comprimento de hipocótilo e raiz. Posteriormente estas partes foram seccionadas e acondicionadas separadamente em sacos de papel fino devidamente identificados, os quais foram postos para secar em estufa a $60^{\circ} \mathrm{C}$ por 48 horas. Após este prazo os materiais foram deixados esfriar em dessecador e pesados em balança analítica com precisão de 0,001g (Nakagawa, 1999).

Nos experimentos o delineamento adotado foi inteiramente casualisado num esquema fatorial $4 \times 5$, entre cultivares e concentrações de alumínio e cultivares e diferentes valores de $\mathrm{pH}$, com quatro repetições. Os valores de porcentagem de germinação, plântulas anormais e classificação de vigor foram transformados pela fórmula arcsen da raiz quadrada $(x / 100)$. Os dados foram tabulados e analisados estatisticamente com auxílio do software SANEST (Zonta et al., 1984), sendo utilizados o teste $\mathrm{F}$ para análise de variância e o teste de Tukey para a comparação das médias.

\section{RESULTADOS E DISCUSSÃO}

$\mathrm{Na}$ Tabela 1 tem-se os resultados de porcentagem de germinação e de plântulas anormais em função de diferentes cultivares e concentrações de alumínio durante a germinação. Não houve diferença estatística entre as médias dos tratamentos e cultivares, os quais comportaram-se homogeneamente com relação a germinação. Souza Filho \& Dutra (1998) trabalhando

Tabela 1 - Porcentagem de germinação e de plântulas anormais de quatro cultivares de soja (Glycine max (L.) Merrill) expostos a concentrações de alumínio durante a germinação. Presidente Prudente, 1999.

\begin{tabular}{|c|c|c|c|c|c|}
\hline \multirow{2}{*}{ Alumínio } & \multicolumn{5}{|c|}{ Cultivar } \\
\hline & Pioneira & Conquista & Xingu & Pintado & Média \\
\hline \multirow[t]{2}{*}{$\mathrm{mmol}_{\mathrm{c}} \mathrm{dm}^{-3}$} & ------------------- & -------------- & - & -----------. & ------------- \\
\hline & \multicolumn{5}{|c|}{ Germinação } \\
\hline 0 & 90 & 84 & 83 & 84 & $85 \mathrm{~A}$ \\
\hline 0,25 & 92 & 92 & 79 & 87 & $87 \mathrm{~A}$ \\
\hline 0,5 & 92 & 85 & 84 & 88 & $87 \mathrm{~A}$ \\
\hline 1 & 90 & 87 & 88 & 87 & $88 \mathrm{~A}$ \\
\hline 1,5 & 72 & 84 & 84 & 92 & $83 \mathrm{~A}$ \\
\hline \multirow[t]{2}{*}{ Média } & $87 \mathrm{a}$ & $86 \mathrm{a}$ & $84 \mathrm{a}$ & $88 \mathrm{a}$ & \\
\hline & \multicolumn{5}{|c|}{ Plântula Anormal } \\
\hline 0 & $6 \mathrm{aA}$ & $9 \mathrm{aA}$ & $12 \mathrm{aA}$ & $12 \mathrm{aA}$ & 10 \\
\hline 0,25 & $5 \mathrm{aA}$ & $4 \mathrm{aA}$ & $10 \mathrm{aAB}$ & $9 \mathrm{aA}$ & 7 \\
\hline 0,5 & $2 \mathrm{aAB}$ & $6 \mathrm{aA}$ & $8 \mathrm{aAB}$ & $7 \mathrm{aA}$ & 5 \\
\hline 1 & $8 \mathrm{aA}$ & 8 aA & $3 \mathrm{aB}$ & 5 aA & 6 \\
\hline 1,5 & $0 \mathrm{bB}$ & $9 \mathrm{aA}$ & $7 \mathrm{aAB}$ & $4 \mathrm{aA}$ & 4 \\
\hline Média & 3 & 7 & 8 & 8 & \\
\hline
\end{tabular}

Letras distintas, maiúsculas nas colunas e minúsculas nas linhas, indicam diferença significativa no teste Tukey com $5 \%$.

Scientia Agricola, v.59, n.1, p.145-153, jan./mar. 2002 
com calopogônio não encontraram efeito de concentração de $\mathrm{Al}^{3+}$ abaixo de $20 \mathrm{mmol}_{\mathrm{c}} \mathrm{dm}^{-3} \mathrm{na}$ germinação. Szymanska \& Molas (1996) trabalharam com germinação de melão em concentrações de $\mathrm{Al}^{3+}$ que variaram de 1 a $40 \mathrm{mg} \mathrm{dm}^{-3}\left(0,12\right.$ a $\left.4,5 \mathrm{mmol}_{\mathrm{c}} \mathrm{dm}^{-3}\right)$ não encontrando efeito na germinação.

$\mathrm{Na}$ avaliação de plântulas anormais, ocorreu interação significativa entre os efeitos dos fatores cultivares e concentrações de alumínio, porém os resultados demonstraram que, os cultivares apresentaram um maior número de plântulas anormais nas menores concentrações de alumínio, tendo estes valores decrescidos com o aumento da dose, pois na dose mais elevada ocorreu uma maior mortalidade das sementes.

$\mathrm{Na}$ Tabela 2 observam-se os resultados de comprimento de hipocótilo e raiz em função dos diferentes cultivares e concentrações de alumínio. $O$ comprimento de hipocótilo foi afetado de forma independente. O tratamento com água pura, ou seja, zero de concentração de alumínio, foi o que produziu menor comprimento de hipocótilo, apresentando um sinergismo aparente entre esta variável e baixas concentrações de alumínio, pois a concentração de alumínio de $0,25 \mathrm{mmol}_{\mathrm{c}} \mathrm{dm}^{-3}$ foi a que produziu o maior comprimento de hipocótilo, diferenciando-se contudo, apenas de água pura. Os cultivares 'Xingu' e 'Pintado' foram os que apresentaram maior comprimento de hipocótilo, seguidos pelo cultivar 'Pioneira' e por 'Conquista' que apesar de apresentar o pior desempenho não se diferenciou de 'Pioneira'. Estes dados corroboram Szymanska \& Molas (1996) que observaram que em concentrações de $\mathrm{Al}^{3+}$ na faixa de $1 \mathrm{a} 5 \mathrm{mg} \mathrm{dm}^{-3}$, ou seja, 0,12 a $0,5 \mathrm{mmol}_{\mathrm{c}} \mathrm{dm}^{-3}$ o crescimento da plântula de melão foi estimulado, não acarretando anormalidades.

Para comprimento de raiz, ocorreu uma interação significativa entre os fatores cultivares e concentrações de alumínio. $O$ tratamento com concentração de alumínio de $1,0 \mathrm{mmol}_{\mathrm{c}} \mathrm{dm}^{-3}$, foi o que produziu maior comprimento de raiz, seguido pelo tratamento de concentração de alumínio de $0,25 \mathrm{mmol}_{\mathrm{c}} \mathrm{dm}^{-3}$. Todos os cultivares apresentaram um aumento no comprimento de raiz quando submetidos ao tratamento com alumínio. Porém o cultivar 'Conquista' que apresentou um menor comprimento de hipocótilo, também foi o que produziu um menor comprimento de raiz. A avaliação pelo comprimento radicular foi eficiente para separar genótipos de soja submetidas a 0,17 e 0,34 $\mathrm{mmol}_{\mathrm{c}} \mathrm{Al}^{3+}$ (Spehar \& Makita, 1994), porém, neste trabalho, a melhor concentração para tal finalidade foi de $1,0 \mathrm{mmol}_{\mathrm{c}} \mathrm{dm}^{-3}$ onde também ocorreram as maiores médias de crescimento radicular.

Os fatores afetaram de forma independente o peso seco de parte aérea (Tabela 3). A concentração de alumínio de $0,5 \mathrm{mmol}_{\mathrm{c}} \mathrm{dm}^{-3}$ foi o que produziu o maior peso seco de parte aérea, seguido pelo tratamento com água pura. Os demais tratamentos provocaram uma redução no peso seco de parte aérea. Os cultivares 'Pintado' e 'Pioneira', diferenciaram-se de 'Xingu', não havendo diferenças entre estes e o cultivar 'Conquista'.

No peso seco de raiz, não houve diferenças entre os cultivares, podendo-se observar o mesmo em relação aos tratamentos, onde os cultivares se apresentaram de forma homogênea.

Tabela 2 - Comprimento de hipocótilo e de raiz de quatro cultivares de soja (Glycine max (L.) Merrill) expostos a concentrações de alumínio durante a germinação. Presidente Prudente, 1999.

\begin{tabular}{|c|c|c|c|c|c|}
\hline \multirow{2}{*}{ Alumínio } & \multicolumn{5}{|c|}{ Cultivar } \\
\hline & Pioneira & Conquista & Xingu & Pintado & Média \\
\hline \multirow[t]{2}{*}{$\mathrm{mmol}_{\mathrm{c}} \mathrm{dm}^{-3}$} & \multicolumn{5}{|c|}{ 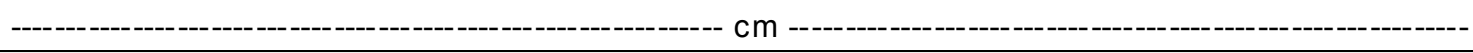 } \\
\hline & \multicolumn{5}{|c|}{ Hipocótilo } \\
\hline 0 & 4,72 & 5,41 & 6,19 & 6,84 & $5,79 \mathrm{~B}$ \\
\hline 0,25 & 7,16 & 6,92 & 7,64 & 7,15 & $7,22 \mathrm{~A}$ \\
\hline 0,5 & 6,95 & 5,28 & 6,74 & 6,28 & $6,31 \mathrm{AB}$ \\
\hline 1 & 6,24 & 4,55 & 6,91 & 6,91 & $6,15 \mathrm{AB}$ \\
\hline 1,5 & 5,46 & 7,09 & 6,63 & 6,94 & $6,53 \mathrm{AB}$ \\
\hline \multirow[t]{2}{*}{ Média } & $6,11 \mathrm{ab}$ & $5,85 \mathrm{~b}$ & $6,82 \mathrm{a}$ & $6,82 \mathrm{a}$ & \\
\hline & \multicolumn{5}{|c|}{ Raiz } \\
\hline 0 & $2,91 \mathrm{aC}$ & $3,33 \mathrm{aB}$ & $4,53 \mathrm{aB}$ & $3,43 \mathrm{aB}$ & 3,55 \\
\hline 0,25 & $5,01 \mathrm{abB}$ & $4,16 \mathrm{bB}$ & $5,96 \mathrm{aB}$ & $4,30 \mathrm{abB}$ & 4,86 \\
\hline 0,5 & $4,24 \mathrm{abBC}$ & $2,94 \mathrm{bB}$ & $4,70 \mathrm{aB}$ & $3,30 \mathrm{abB}$ & 3,79 \\
\hline 1 & 9,10 aA & $7,25 \mathrm{bA}$ & $8,95 \mathrm{abA}$ & $10,00 \mathrm{aA}$ & 8,83 \\
\hline 1,5 & $3,24 \mathrm{aBC}$ & $4,10 \mathrm{aB}$ & $4,93 \mathrm{aB}$ & $3,98 \mathrm{aB}$ & 4,06 \\
\hline Média & 4,90 & 4,36 & 5,82 & 5,00 & \\
\hline
\end{tabular}

Letras distintas, maiúsculas nas colunas e minúsculas nas linhas, indicam diferença significativa no teste Tukey com $5 \%$. 
Ocorreu interação significativa entre os fatores cultivares e concentrações de alumínio (Tabela 4). Dentre os tratamentos utilizados, as concentrações de alumínio de $1,0 \mathrm{mmol}_{\mathrm{c}} \mathrm{dm}^{-3}$ e $1,5 \mathrm{mmol}_{\mathrm{c}} \mathrm{dm}^{-3}$ foram as que apresentaram as menores porcentagens na classificação de vigor. Os demais tratamentos apresentaram valores mais altos na classificação, indicando que essas concentrações afetaram menos o vigor de plântulas quando comparados com as concentrações de 1,0 e 1,5 $\mathrm{mmol}_{\mathrm{c}} \mathrm{dm}^{-3}$. Os cultivares 'Xingu', 'Pioneira' e 'Pintado' apresentaram os maiores valores na classificação de vigor na maioria dos tratamentos. O cultivar 'Conquista' produziu os menores valores na classificação de vigor na maioria dos tratamentos.

As concentrações de alumínio não afetaram a germinação e o peso seco de raiz, em contrapartida o comprimento de hipocótilo, o comprimento de raiz, o peso seco de parte aérea e a classificação de vigor foram afetados por ambos os fatores. O comprimento de raiz teve seu maior desenvolvimento no tratamento com concentração de alumínio de $1,0 \mathrm{mmol}_{\mathrm{c}} \mathrm{dm}^{-3}$, e o peso seco de parte aérea foi numericamente maior no tratamento com concentração de alumínio de $0,5 \mathrm{mmol}$ $\mathrm{dm}^{-3}$. Estes dados reforçam o que Szymanska \& Molas (1996) observaram, indicando que concentrações baixas de $\mathrm{Al}^{3+}$ podem até estimular o desenvolvimento inicial do vegetal. Para os autores, trabalhando com melão, a faixa onde houve o estímulo foi de 1 a $5 \mathrm{mg} \mathrm{dm}^{-3}(0,12$ a 0,5 $\left.\mathrm{mmol}_{\mathrm{c}} \mathrm{dm}^{-3}\right)$ de $\mathrm{Al}^{3+}$ na solução. Em soja a faixa onde ocorreu o estímulo foi entre 0,25 e $1 \mathrm{mmol}_{\mathrm{c}} \mathrm{dm}^{-3}$. $\mathrm{Na}$ solução do solo, de um modo geral, a literatura aponta que menos de $5 \mathrm{mmol}_{\mathrm{c}} \mathrm{dm}^{-3}$ de $\mathrm{Al}^{3+}$ não deve ser tóxico; $5-10 \mathrm{mmol}_{\mathrm{c}} \mathrm{dm}^{-3}$ é provavelmente tóxico e $10 \mathrm{mmolc} \mathrm{dm}^{3}$ tem alta probabilidade de ser, pois as espécies e os cultivares dentro da mesma espécie podem mostrar tolerância muito diferente à toxidez de alumínio (Malavolta, 1980). Parece que no início do desenvolvimento as plântulas se mostram muito mais sensíveis, pois em soja,

Tabela 3 - Peso seco de parte aérea e de raiz de quatro cultivares de soja (Glycine max (L.) Merrill) expostos a concentrações de alumínio durante a germinação. Presidente Prudente, 1999.

\begin{tabular}{|c|c|c|c|c|c|}
\hline \multirow{2}{*}{ Alumínio } & \multicolumn{5}{|c|}{ Cultivar } \\
\hline & Pioneira & Conquista & Xingu & Pintado & Média \\
\hline \multirow[t]{2}{*}{$\mathrm{mmol}_{\mathrm{c}} \mathrm{dm}^{-3}$} & ---------------- & --------------- & $---g$----. & ------------- & 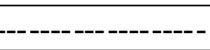 \\
\hline & \multicolumn{5}{|c|}{ Parte Aérea } \\
\hline 0 & 0,22 & 0,21 & 0,18 & 0,23 & $0,21 \mathrm{~B}$ \\
\hline 0,25 & 0,21 & 0,15 & 0,16 & 0,17 & $0,17 \mathrm{C}$ \\
\hline 0,5 & 0,29 & 0,25 & 0,21 & 0,32 & $0,27 \mathrm{~A}$ \\
\hline 1 & 0,17 & 0,18 & 0,17 & 0,19 & $0,18 \mathrm{C}$ \\
\hline 1,5 & 0,16 & 0,17 & 0,14 & 0,18 & $0,16 \mathrm{C}$ \\
\hline \multirow[t]{2}{*}{ Média } & $0,21 \mathrm{a}$ & $0,19 a b$ & $0,17 \mathrm{~b}$ & $0,22 a$ & \\
\hline & \multicolumn{5}{|c|}{ Raiz } \\
\hline 0 & 0,028 & 0,018 & 0,020 & 0,023 & $0,022 \mathrm{~A}$ \\
\hline 0,25 & 0,028 & 0,015 & 0,018 & 0,013 & $0,018 \mathrm{~A}$ \\
\hline 0,5 & 0,043 & 0,028 & 0,065 & 0,025 & $0,040 \mathrm{~A}$ \\
\hline 1 & 0,023 & 0,025 & 0,013 & 0,020 & $0,020 \mathrm{~A}$ \\
\hline 1,5 & 0,048 & 0,023 & 0,010 & 0,013 & $0,023 \mathrm{~A}$ \\
\hline Média & $0,034 a$ & $0,022 a$ & $0,025 a$ & $0,019 a$ & \\
\hline
\end{tabular}

Letras distintas, maiúsculas nas colunas e minúsculas nas linhas, indicam diferença significativa no teste Tukey com $5 \%$.

Tabela 4 - Classificação de vigor de plântulas de quatro cultivares de soja (Glycine max (L.) Merrill) expostos a concentrações de alumínio durante a germinação. Presidente Prudente, 1999.

\begin{tabular}{|c|c|c|c|c|c|}
\hline \multirow{2}{*}{ Alumínio } & \multicolumn{5}{|c|}{ Cultivar } \\
\hline & Pioneira & Conquista & Xingu & Pintado & Média \\
\hline $\mathrm{mmol}_{\mathrm{c}} \mathrm{dm}^{-3}$ & ------------------ & -------------. & --- \% --- & ----------- & 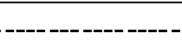 \\
\hline 0 & 36 abAB & $30 \mathrm{abA}$ & $38 \mathrm{aA}$ & $23 \mathrm{bAB}$ & 31 \\
\hline 0,25 & $42 \mathrm{aA}$ & $22 \mathrm{bAB}$ & 29 abAB & $39 \mathrm{aA}$ & 33 \\
\hline 0,5 & $43 \mathrm{aA}$ & $15 \mathrm{bBC}$ & $32 \mathrm{aAB}$ & $36 \mathrm{aA}$ & 31 \\
\hline 1 & $22 \mathrm{aBC}$ & $15 \mathrm{aBC}$ & $18 \mathrm{aB}$ & $19 \mathrm{aB}$ & 18 \\
\hline 1,5 & $14 \mathrm{abC}$ & $8 \mathrm{bC}$ & $22 \mathrm{aB}$ & $12 \mathrm{abB}$ & 14 \\
\hline Média & 31 & 17 & 27 & 25 & \\
\hline
\end{tabular}

Letras distintas, maiúsculas nas colunas e minúsculas nas linhas, indicam diferença significativa no teste Tukey com $5 \%$.

Scientia Agricola, v.59, n.1, p.145-153, jan./mar. 2002 
neste trabalho, concentrações acima de $1 \mathrm{mmol}_{\mathrm{c}} \mathrm{dm}^{-3}$ já afetaram o desenvolvimento da plântula. Em melão concentrações acima de $20 \mathrm{mg} \mathrm{dm}^{-3}\left(2,1 \mathrm{mmol}_{\mathrm{c}} \mathrm{dm}^{-3}\right)$ provocaram inibição do crescimento da plântula e danos morfológicos (Szymanska \& Molas, 1996).

Uma explicação para a redução do desenvolvimento radicular seria que $\circ \mathrm{Al}^{3+}$ reagiria com cadeias de ácido poligalacturônico das paredes das células jovens dando compostos pécticos "errados" (substituição ou deslocamento de $\mathrm{Ca}$ ) do que resultaria a perda de elasticidade celular. A região meristemática da ponta da raiz mostra um número anormalmente alto de células com dois núcleos, o que indicaria paralisação do processo de divisão celular (Malavolta, 1980). Szymanska \& Molas (1996) observaram que em concentrações de 20 a $40 \mathrm{mg} \mathrm{dm}^{-3}\left(2,1 \mathrm{a} 4,5 \mathrm{mmol}_{\mathrm{c}} \mathrm{dm}^{-3}\right)$ o Al foi encontrado em todas as camadas da raiz e, nas células, estava situado na parede, núcleo e nucléolo. $\mathrm{Na}$ concentração mais alta $\left(40 \mathrm{mg} \mathrm{dm}^{-3}\right)$ foi encontrado no citoplasma das células.

Os cultivares apresentaram comportamentos distintos em função das concentrações de alumínio. Os cultivares 'Xingu', 'Pintado' e 'Pioneira' se destacaram em todos os tratamentos principalmente em comprimento de hipocótilo ('Xingu' e 'Pintado'), comprimento de raiz ('Xingu', 'Pintado' e 'Pioneira'), peso seco de parte aérea ('Pintado' e 'Pioneira') e classificação de vigor ('Xingu' e 'Pioneira'). Segundo Blaha et al. (1997) os cultivares podem apresentar diferenças quanto submetidos a condições ácidas de toxidez de $\mathrm{Al}^{3+}$ e o uso de seleção na fase juvenil, para estas condições de desenvolvimento, parece ser favorável.

As concentrações de alumínio não afetaram a germinação e o peso seco de raiz, em contrapartida o comprimento de hipocótilo e de raiz, o peso seco de parte aérea e a classificação de vigor foram afetados. Na faixa entre 0,25 e $1 \mathrm{mmol}_{\mathrm{c}} \mathrm{dm}^{-3}$ ocorreu estímulo para o desenvolvimento da plântula (comprimento de hipocótilo e raiz). Estatisticamente, o comprimento de raiz teve seu maior desenvolvimento no tratamento de $1,0 \mathrm{mmolc} \mathrm{Al}^{3+}$ $\mathrm{dm}^{3}$. Os cultivares 'Xingu', 'Pintado' e 'Pioneira' se destacaram em todos os tratamentos, sendo que 'Conquista' apresentou uma tendência para maior sensibilidade aos tratamentos de $\mathrm{Al}^{3+}$. Todavia, altas concentrações de alumínio ( $92 \%$ de saturação de alumínio) afetam o crescimento radicular (Bushamuka \& Zobel, 1998) e segundo Rutty et al. (1995) concentrações de apenas $0,132 \mathrm{mmol}_{\mathrm{c}} \mathrm{dm}^{-3}$ seriam suficientes para afetar o desenvolvimento de plântulas, o que não ocorreu neste trabalho.

Os fatores afetaram independentemente a germinação (Tabela 5). O pH 6,0 apresentou o maior valor numérico de germinação não se diferenciando estatisticamente dos tratamentos 7,0,5,5 e 5,0. Estes não diferiram do $\mathrm{pH} 6,0$ e nem de $\mathrm{pH} 4,5 . \mathrm{O} \mathrm{pH} 4,5$ produziu o menor valor de germinação. O cultivar 'Xingu' obteve a maior média de germinação, seguido pelos cultivares
'Pioneira' e 'Pintado'. Estes não diferiram do cultivar 'Xingu' e também não diferiram do 'Conquista' o qual obteve o menor valor de germinação. Souza Filho \& Dutra (1998) reportaram para calopogônio a não interferência do pH na germinação em pH variando de 3 a 11 .

Os resultados de plântulas anormais não foram afetados pelos tratamentos de $\mathrm{pH}$. Os cultivares que produziram maior germinação foram aqueles que apresentaram menores valores de plântulas anormais.

Os fatores afetaram os comprimentos od hipocótilo e da raiz de forma independente (Tabela 6). A água pura $(\mathrm{pH} 7,0)$ foi o tratamento que produziu maior comprimento de hipocótilo. Os demais tratamentos provocaram redução no crescimento do hipocótilo das plântulas.

Os cultivares se comportaram de forma homogênea em relação ao comprimento de hipocótilo, tanto que essa medida não os diferiu estatisticamente. Quanto ao crescimento de raiz, os tratamentos com $\mathrm{pH}$ 7,0 e $\mathrm{pH} 6,0$ produziram maior comprimento de raiz. Os tratamentos de $\mathrm{pH} 5,5,5,0$ e 4,5 afetaram significativamente as plântulas reduzindo o crescimento das raízes. O cultivar 'Conquista' apresentou menores valores de comprimento de raiz. Davis et al. (1993) demonstraram para Pennisetum glaucum que o valor de $\mathrm{pH}$ variando de 5 a 7 não afetou o desenvolvimento de parte aérea, enquanto que os mesmos valores foram os melhores para o desenvolvimento das raízes, sendo que em valores menores de $\mathrm{pH}$ o crescimento de raízes foi comprometido.

Os fatores afetaram ambos os pesos secos de forma independente (Tabela 7). $\mathrm{O}$ pH 7,0 foi o que produziu maior peso seco de parte aérea. Os demais tratamentos provocaram redução no peso seco de parte aérea de forma significativa, sendo o menor valor encontrado em $\mathrm{pH} 4,5$. O cultivar 'Pintado' obteve os valores mais altos de peso seco de parte aérea, não se diferenciando apenas do cultivar 'Conquista', o qual além de apresentar os menores valores de comprimento de raiz, também apresentou menores pesos secos de parte aérea e raiz. Os cultivares 'Xingu' e 'Pioneira' apresentaram uma significativa redução de peso seco de parte aérea quando $0 \mathrm{pH}$ baixou de 5,0 para 4,5 mostrando uma grande sensibilidade ao estresse ácido. $\mathrm{O}$ peso seco de raiz foi maior no $\mathrm{pH} 7,0$ diferenciado estatisticamente dos valores de $\mathrm{pH}$ de 5,5, 5,0 e 4,5. Os demais tratamentos reduziram significativamente o peso seco da raiz. O cultivar 'Pintado' diferenciou-se apenas do cultivar 'Conquista', não havendo diferenças estatísticas entre estes e os outros dois cultivares.

Ocorreu interação significativa entre os fatores cultivares e pH (Tabela 8). O cultivar 'Pioneira' não foi afetado pelos tratamentos de $\mathrm{pH}$, entretanto todos os outros o foram, sendo que o cultivar 'Conquista' apresentou os menores valores em $\mathrm{pH} 4,5$, diferindo dos cultivares 'Pioneira' e 'Xingu'. Numericamente, 'Xingu' apresentou-se como tendo melhor vigor. 
Tabela 5 - Porcentagem de Germinação e de plântulas anormais de quatro cultivares de soja (Glycine max (L.) Merrill) expostos a valores de pH durante a germinação. Presidente Prudente, 1999.

\begin{tabular}{|c|c|c|c|c|c|}
\hline \multirow{2}{*}{ Valor de $\mathrm{pH}$} & \multicolumn{5}{|c|}{ Cultivar } \\
\hline & Pioneira & Conquista & Xingu & Pintado & Média \\
\hline & \multicolumn{5}{|c|}{------------------------------------------------------------ $\%$} \\
\hline & \multicolumn{5}{|c|}{ Germinação } \\
\hline 7 & 91 & 89 & 97 & 93 & $92 \mathrm{AB}$ \\
\hline 6 & 96 & 93 & 99 & 92 & $95 \mathrm{~A}$ \\
\hline 5,5 & 95 & 93 & 94 & 93 & $94 \mathrm{AB}$ \\
\hline 5 & 92 & 89 & 94 & 90 & $91 \mathrm{AB}$ \\
\hline 4,5 & 93 & 76 & 93 & 89 & 88 B \\
\hline \multirow[t]{2}{*}{ Média } & $93 a b$ & $88 \mathrm{~b}$ & $95 \mathrm{a}$ & $91 a b$ & \\
\hline & \multicolumn{5}{|c|}{ Plântula Anormal } \\
\hline 7 & 1 & 7 & 2 & 2 & $3 \mathrm{~A}$ \\
\hline 6 & 2 & 2 & 1 & 5 & $2 \mathrm{~A}$ \\
\hline 5,5 & 3 & 4 & 3 & 3 & $3 \mathrm{~A}$ \\
\hline 5 & 5 & 5 & 4 & 5 & $5 \mathrm{~A}$ \\
\hline 4,5 & 3 & 10 & 3 & 4 & $5 \mathrm{~A}$ \\
\hline Média & $3 \mathrm{~b}$ & $6 a$ & $3 \mathrm{~b}$ & $4 a b$ & \\
\hline
\end{tabular}

Letras distintas, maiúsculas nas colunas e minúsculas nas linhas, indicam diferença significativa no teste Tukey a $5 \%$.

Tabela 6 - Comprimento de hipocótilo e de raiz de quatro cultivares de soja (Glycine max (L.) Merrill) expostos a valores de pH durante a germinação. Presidente Prudente, 1999.

\begin{tabular}{|c|c|c|c|c|c|}
\hline \multirow{2}{*}{ Valor de $\mathrm{pH}$} & \multicolumn{5}{|c|}{ Cultivar } \\
\hline & Pioneira & Conquista & Xingu & Pintado & Média \\
\hline & \multicolumn{5}{|c|}{ 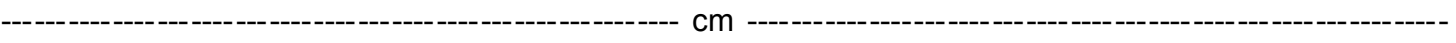 } \\
\hline & \multicolumn{5}{|c|}{ Hipocótilo } \\
\hline 7 & 6,84 & 9,78 & 6,87 & 7,98 & $7,87 \mathrm{~A}$ \\
\hline 6 & 4,48 & 3,37 & 4,83 & 5,26 & $4,48 \mathrm{~B}$ \\
\hline 5,5 & 3,68 & 1,66 & 3,5 & 3,51 & $3,09 \mathrm{~B}$ \\
\hline 5 & 5,07 & 1,89 & 4,26 & 5,58 & $4,20 \mathrm{~B}$ \\
\hline 4,5 & 1,74 & 2,61 & 2,83 & 4,16 & $2,83 \mathrm{~B}$ \\
\hline \multirow[t]{2}{*}{ Média } & $4,36 \mathrm{a}$ & $3,86 a$ & $4,46 \mathrm{a}$ & $5,30 a$ & \\
\hline & \multicolumn{5}{|c|}{ Raiz } \\
\hline 7 & 3,77 & 3,87 & 4,66 & 3,93 & $4,06 \mathrm{~A}$ \\
\hline 6 & 2,75 & 1,71 & 3,35 & 2,73 & $2,63 \mathrm{~B}$ \\
\hline 5,5 & 2,53 & 1,00 & 2,81 & 2,2 & $2,13 \mathrm{~B}$ \\
\hline 5 & 2,2 & 1,00 & 2,78 & 2,23 & $2,05 \mathrm{~B}$ \\
\hline 4,5 & 1,28 & 1,83 & 2,81 & 2,18 & $2,02 \mathrm{~B}$ \\
\hline Média & $2,51 \mathrm{ab}$ & $1,88 \mathrm{~b}$ & 3,28 a & 2,65 a & \\
\hline
\end{tabular}

Letras distintas, maiúsculas nas colunas e minúsculas nas linhas, indicam diferença significativa no teste Tukey a $5 \%$.

Os tratamentos de $\mathrm{pH}$ afetaram a germinação, o comprimento de hipocótilo e de raiz, o peso seco de parte aérea e de raiz e a classificação do vigor. A germinação foi maior numericamente no $\mathrm{pH}$ 6,0 enquanto que $\mathrm{o} \mathrm{pH} 7,0$ produziu maiores comprimentos de hipocótilo e de raiz, maiores pesos secos de parte aérea e de raiz, corroborando os dados de Bokert \& Cox (1999) e Fageria \& Baligar (1999)
Os cultivares apresentaram comportamentos distintos em função dos tratamentos de $\mathrm{pH}$. O cultivar 'Xingu' apresentou um resultado numericamente superior aos demais. 'Pioneira' apresentou um desempenho médio e 'Pintado' e 'Conquista' inferiores mesmo nos tratamentos menos ácidos, indicando que a germinação foi um evento pouco afetado pela acidez do substrato conforme observado por Souza Filho \& Dutra (1998). 
Tabela 7 - Peso seco de parte aérea e de raiz de quatro cultivares de soja (Glycine max (L.) Merrill) expostos valores de pH durante a germinação. Presidente Prudente, 1999.

\begin{tabular}{|c|c|c|c|c|c|}
\hline \multirow{2}{*}{ Valor de $\mathrm{pH}$} & \multicolumn{5}{|c|}{ Cultivar } \\
\hline & Pioneira & Conquista & Xingu & Pintado & Média \\
\hline & \multicolumn{5}{|c|}{ - } \\
\hline & \multicolumn{5}{|c|}{ Parte aérea } \\
\hline 7 & 0,160 & 0,140 & 0,130 & 0,150 & $0,145 \mathrm{~A}$ \\
\hline 6 & 0,090 & 0,060 & 0,080 & 0,110 & $0,085 \mathrm{~B}$ \\
\hline 5,5 & 0,070 & 0,020 & 0,060 & 0,080 & $0,057 \mathrm{BC}$ \\
\hline 5 & 0,110 & 0,040 & 0,090 & 0,110 & 0,087 B \\
\hline 4,5 & 0,020 & 0,040 & 0,040 & 0,090 & $0,047 \mathrm{C}$ \\
\hline \multirow[t]{2}{*}{ Média } & $0,090 \mathrm{ab}$ & $0,060 \mathrm{~b}$ & $0,080 \mathrm{ab}$ & 0,108 a & \\
\hline & \multicolumn{5}{|c|}{ Raiz } \\
\hline 7 & 0,010 & 0,020 & 0,010 & 0,010 & $0,012 \mathrm{~A}$ \\
\hline 6 & 0,010 & 0,006 & 0,010 & 0,010 & $0,009 A B$ \\
\hline 5,5 & 0,010 & 0,003 & 0,010 & 0,010 & 0,008 B \\
\hline 5 & 0,010 & 0,007 & 0,010 & 0,020 & $0,011 \mathrm{~B}$ \\
\hline 4,5 & 0,010 & 0,010 & 0,007 & 0,010 & 0,009 B \\
\hline Média & $0,010 a b$ & $0,009 \mathrm{~b}$ & $0,009 \mathrm{ab}$ & $0,012 a$ & \\
\hline
\end{tabular}

Letras distintas, maiúsculas nas colunas e minúsculas nas linhas, indicam diferença significativa no teste Tukey a $5 \%$.

Tabela 8 - Classificação de vigor de plântulas de quatro cultivares de soja (Glycine max (L.) Merrill) expostos valores de pH durante a germinação. Presidente Prudente, 1999.

\begin{tabular}{cccccc}
\hline \multirow{2}{*}{ Valor de pH } & \multicolumn{5}{c}{ Cultivar } \\
\cline { 2 - 5 } & Pioneira & Conquista & Xingu & Pintado & Média \\
\hline 7 & $46 \mathrm{abA}$ & $24 \mathrm{cAB}$ & $63 \mathrm{aA}$ & $28 \mathrm{bcA}$ & 40 \\
\hline 6 & $38 \mathrm{bA}$ & $27 \mathrm{bAB}$ & $63 \mathrm{aA}$ & $34 \mathrm{bA}$ & 41 \\
5,5 & $30 \mathrm{bcA}$ & $43 \mathrm{abA}$ & $55 \mathrm{aAB}$ & $23 \mathrm{cA}$ & 37 \\
5 & $32 \mathrm{aA}$ & $27 \mathrm{aAB}$ & $38 \mathrm{aB}$ & $20 \mathrm{aA}$ & 29 \\
4,5 & $40 \mathrm{aA}$ & $14 \mathrm{bB}$ & $38 \mathrm{aB}$ & $36 \mathrm{aA}$ & 31 \\
\hline Média & 37 & 27 & 51 & 28 & \\
\hline
\end{tabular}

Letras distintas, maiúsculas nas colunas e minúsculas nas linhas, indicam diferença significativa no teste Tukey a $5 \%$.

O cultivar 'Conquista' foi o mais sensível aos tratamentos de alta acidez. As variáveis responderam similarmente aos dados exibidos por Obroucheva \& Antipova (1994), onde houve o crescimento celular em ambiente ácido, todavia este crescimento pode ser limitado por valores próximos ou abaixo de $\mathrm{pH}$ 4,0 (Davis et al., 1993; Rutty et al., 1995; Bushamuka \& Zobel, 1998). Chukwuma \& Tiwari (1995) conseguiram separar 22 de 85 genótipos de soja em ensaios trabalhando com acidez média ( $\mathrm{pH}$ entre 5,0 e 5,5).

Os tratamentos de $\mathrm{pH}$ afetaram a germinação, o comprimento de hipocótilo e de raiz, o peso seco de parte aérea e de raiz e a classificação do vigor. A germinação foi maior numericamente no $\mathrm{pH}$ 6,0 enquanto que o $\mathrm{pH} 7,0$ produziu maior comprimento de hipocótilo, de raiz, maior peso seco de parte aérea e de raiz. 0 cultivar 'Xingu', se destacou por apresentar os maiores valores numéricos de germinação mesmo nos tratamentos com maior acidez, indicando que a germinação foi um evento pouco afetado pelos tratamentos. O cultivar 'Conquista' foi o mais sensível aos tratamentos de alta acidez.

\section{AGRADECIMENTO}

À Sementes Maggi Ltda pela cessão do germoplasma utilizado neste trabalho.

\section{REFERÊNCIAS BIBLIOGRÁFICAS}

BEYROUTY, C.A.; KEINO, J.K.; GBUR, E.E.; HANSON, M.G. Phytoxic concentrations of subsoil aluminum as influenced by soils and landascape position. Soil Science, v.165, p.135143, 2000.

BLAHA, L.; DVORAK, M.; PECHOVA, M.; HROZINKOVA, A. Influence of low $\mathrm{pH}$ and aluminium ions on the nutrient uptake and growth in juvenile phase of cereal cultivars. Scientia Agriculturae Bohemica, v.28, p.81-94, 1997. 
BORKERT, C.M.; COX, F.R. Effects of acidity at high soil zinc, copper and manganese on peanut, rice and soybean. Communications in Soil Science and Plant Analysis, v.30, p.1371-1384, 1999.

BRASIL. Ministério da Agricultura e da Reforma Agrária. Regras para análise de Sementes. Brasília: SNDA; DNDDV; CLAV, 1992. 365p.

BRASIL. Ministério da Agricultura. Recomendações técnicas para a cultura da soja na região central do Brasil. Londrina, 1998. $44 p$

BUERKERT, A.; MARSCHNER, H. Calcium and temperature effects on seedling exudation and root infection of common bean on an acid sandy soil. Plant and Soil, v.147, p.293-303, 1992.

BUSHAMUKA, V.N.; ZOBEL, R.N. Maize and soybean tap, basal and lateral root responses to a stratified acid, aluminum-toxic soil. Crop Science, v.38, p.416-421, 1998.

CHUKWUMA, F.O.; TIWARI, S.C. Screening soybean lines based on phosphorus/aluminum ratio and harvest index values. Journal of the Mississipi Academy of Sciences, v.40, p.1213, 1995.

DAVIS, J.G.; HOSSNER, L.R.; PARSAUD, N. Elemental toxicity effects on germination and growth of pearl millet seedlings. Journal of Plant Nutrition, v.16, p.1957-1968, 1993.

FAGERIA, N.K.; BALIGAR,V.C. Growth and nutrient concentrations of commom bean, lowland rice, corn, soybean and wheat at different soil $\mathrm{pH}$ on an Inceptisol. Journal of Plant Nutrition, v.22, p.1495-1507, 1999.

KRZYZANOWSKI, F.C. Teste de comprimento de raiz de plântulas de soja. Informativo ABRATES, v.2, p.11-14, 1991.

MALAVOLTA, E. Elementos de nutrição mineral de plantas. Piracicaba: Ceres, 1980. 251p.
NAKAGAWA, J. Testes de vigor baseados no desempenho das plântulas. In: KRZYZANOWSKI, F.C.; VIEIRA, R.D.; FRANÇA NETO, J. de B. Vigor de sementes: conceitos e testes. Londrina: ABRATES, 1999. p.2.1-2.21.

OBROUCHEVA, N.V.; ANTIPOVA, O.V. Preparation and initiation of growth in axial organs of germinating quiescent seeds. 2 . initiation of 'acid growth' in the axial organs of broad bean seeds. Russian Journal of Plant Physiology, v.41, p.391395, 1994

RUTTY, T.W.; MACKOWN, C.T.; LAZOF, D.B.; CARTER, T.E. Effects of aluminium on nitrate uptake and assimilation. Plant, Cell and Environment, v.18, p.1325-1331, 1995.

SOUZA FILHO, A. da S.; DUTRA, S. Germination of seeds of Calopogonium mucunoides. Pasturas Tropicales, v.20, p.2630, 1998.

SPEHAR, C.R.; MAKITA, M. Tolerância ao alumínio em plântulas de soja e sua utilização. Pesquisa Agropecuária Brasileira, v.29, p.1927-1932, 1994.

SZYMANSKA, M.; MOLAS, J. The effect of aluminium on early development stages of Cucumis sativus L. Folia Horticulturae, v.8, p.73- 83, 1996.

VITTI, G.C.; CASARIN, V. Calagem e nutrição com enxofre na produtividade da soja. SIMPÓSIO SOBRE CULTURA E PRODUTIVIDADE DA SOJA, 1., Piracicaba, 1991. Anais. Piracicaba: FEALQ, 1992. p.157-179.

ZONTA, E.P.; MACHADO, A.D.; SILVEIRA JR., P. Sistemas de análise estatística para microcomputadores - SANEST. Pelotas: UFPel, 1984. (Registro SEI n06606-0, Categoria $\mathrm{AO})$.

$\overline{\text { Recebido em } 26.06 .00}$ 